\title{
Fibroma uterino em cadela
}

\section{Uterine fibroma in dogs}

\author{
Endrigo Adonis Braga de Araujo, ${ }^{* *}$ Luis Fernando Mercês Chaves Silva, ${ }^{* *}$ Sidnei Nunes de Oliveira, ${ }^{* *}$ Felipe Morales Dalanezi, ${ }^{* \star}$ \\ Leonardo Dourado da Costa, ${ }^{* * *}$ Jéssica Corrêa Rodrigues, ${ }^{* * * *}$ Fabíola Soares Zahn, ${ }^{* *}$ Nereu Carlos Prestes ${ }^{* *}$
}

\begin{abstract}
Resumo
Relatam-se dois casos de fibroma uterino em cadela. Caso 1: cadela Poodle, 15 anos de idade, pesando 3,8 kg, nulípara, apresentando abdômen distendido, anorexia e aquiesia há quatro dias. Aos exames radiográficos e ultrassonográficos visualizou-se neoformação uterina. Caso 2: cadela Poodle de 13 anos de idade, pesando 3,0 kg, nulípara, que à palpação abdominal apresentava uma massa consistente, circular, aparentemente localizada em útero. Ao exame ultrassonográfico da cavidade abdominal, visualizou-se conteúdo uterino e presença de estrutura circular hipoecogênica em útero. Diante dos quadros clínicos, procedeuse ovariosalpingohisterectomia $(\mathrm{OSH})$ de caráter emergencial em ambos os casos e as peças cirúrgicas foram enviadas para realização de exame histopatológico, que revelou como resultado fibroma. Os pacientes retornaram dez dias após o procedimento cirúrgico com a saúde restabelecida.
\end{abstract}

Palavras-chave: canis familiares, patologia, útero, tumor.

\begin{abstract}
Two cases of uterine fibroma in dogs are reported. Case \#1: Poodle bitch, 15 years-old, 3.8kg, nulliparous, presenting distended abdomen, anorexia and aquesia for four days. Radiographic and ultrasonographic examinations evidenced uterine neoformation. Case \#2: Poodle Bitch, 13 years-old, $3.0 \mathrm{~kg}$, nulliparous, abdominal palpation revealed a circular hard mass, apparently involving the uterus. Abdominal ultrassonography evidenced uterine content and the presence of a circular echogenic structure inside the uterus. Emergency ovariosalpingohisterectomy $(\mathrm{OSH})$ was performed in both cases and the diagnosis of uterine fibroma was given by histological examination. Patients returned ten days after the surgical procedure with restored health.
\end{abstract}

Keywords: canis familiares, pathology, uterus, tumor.

\section{Introdução}

As neoplasias do aparelho reprodutor mais comuns em cadelas são as de origem mesenquimal (Souza et al., 2012). Os tumores uterinos representam menos de 0,5 por cento dos tumores do trato reprodutivo em cães e gatos. O mais comum entre eles, que responde por aproximadamente $90 \%$ dos casos, é o Leiomioma, seguido por fibroma, fibroleiomioma e o leiomiossarcoma (Klein, 2001; Maclachlan e Kennedy 2002).

O aparecimento dessas neoplasias pode ser influenciado por hormônios sexuais e frequentemente estão associadas à ocorrência de outras patologias como cistos foliculares ovarianos, tumores secretores de estrógenos, hiperplasia endometrial, hiperplasia e neoplasia mamária (Schlafer e Miller, 2007).

Fibroma é um tumor benigno de consistência firme, brancacento, formato esférico ou ovoide, localizado na parede uterina. Microscopicamente é composto por tecido conjuntivo fibroso (Baba e Câtoi, 2007). A realização da ovariosalpingohisterectomia $(\mathrm{OSH})$ é a terapia mais indicada para neoplasias benignas em útero (McEntee, 2002).

O presente trabalho tem por objetivo descrever dois casos de fibroma uterino em cadela.

\section{Relato de caso}

\section{Caso 1}

Uma cadela, Poodle, de 15 anos de idade, pesando $3,8 \mathrm{~kg}$, nulípara, foi atendida no ambulatório de pequenos animais do setor de reprodução animal da Universidade Estadual Paulista "Júlio de Mesquita Filho" (UNESP)-Botucatu. Apresentava abdômen distendido (Figura 5A), anorexia e aquesia havia quatro dias, polaciúria, não andava havia 1 dia e o último cio havia sido observado aproximadamente um ano antes.

Ao exame físico, apresentava mucosas congestas, desidratação leve, frequência cardíaca e respiratória dentro dos valores normais de referência e à palpação, o abdômen encontravase com consistência firme. Realizou-se exame radiográfico que revelou cavidade abdominal com aspecto homogêneo, abdômen abaulado mais evidente do lado esquerdo, sugerindo neoformação (Figuras 1A e 1B). Ao exame ultrassonográfico da cavidade abdominal, visibilizou-se presença de estrutura heterogênea com áreas cavitárias em topografia de corpo e cornos uterinos, com vascularização ao doppler colorido, deslocando e comprimindo estruturas adjacentes (bexiga, alças intestinais, baço, rins, fígado e estômago) (Figuras 1C e 1D).

${ }^{*}$ Recebido em 14 de julho de 2014 e aceito em 20 de julho de 2015


***Universidade Estadual Paulista "Júlio de Mesquita Filho" Unesp, Departamento de Clínica Veterinária, Botucatu, São Paulo, Brasil.

****Universidade Estadual Paulista “Júlio de Mesquita Filho" Unesp, Departamento de Cirurgia e Anestesiologia Veterinária, Botucatu, São Paulo, Brasil. Autor para correspondência: Nereu Carlos Prestes - nereu@fmvz.unesp.br 

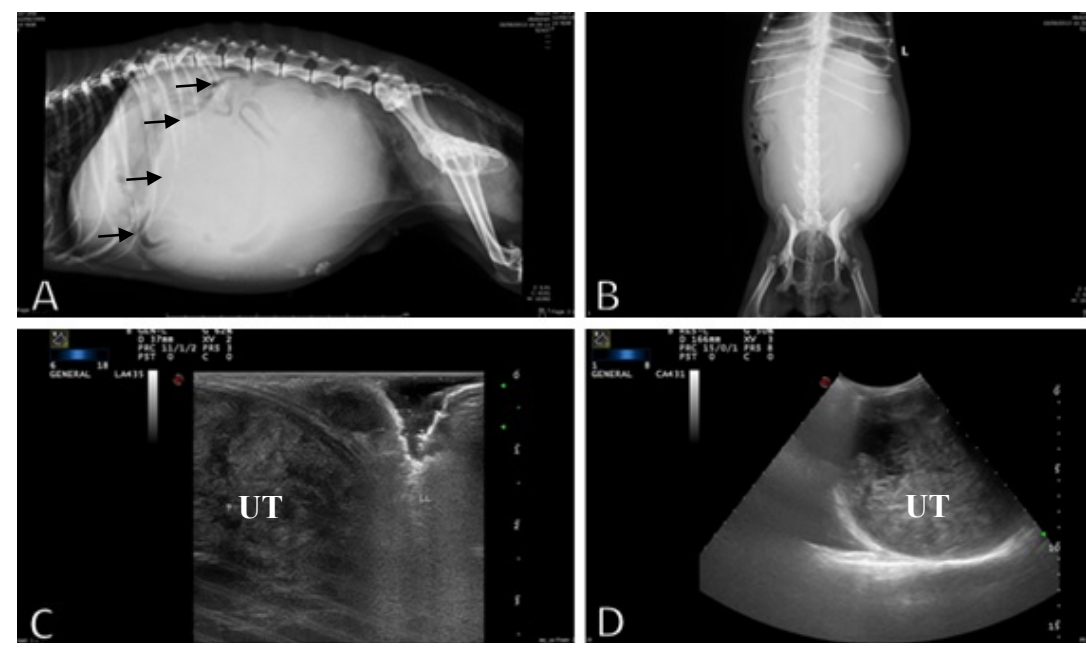

Figura 1A e 1B: Exame radiográfico demonstrando a neoformação (setas). 1C e 1D. Exame ultrassonográfico abdominal apresentando a neoformação em útero.

Efetuou-se colheita de sangue para realização do hemograma e exames bioquímicos, sendo que os achados hematológicos revelaram anemia normocítica e normocrômica, leucograma inflamatório caracterizado por leucocitose grave, neutrofilia com desvio à esquerda e monocitose. O bioquímico apresentou alteração das enzimas hepáticas fosfatase alcalina (FA) e gama glutamil transferase (GGT), além de ureia e creatinina (Tabela 1).

Diante do quadro clínico apresentado, foi realizada laparotomia exploratória de caráter emergencial. Devido ao estado apático do animal, não foi realizada medicação pré-anestésica (MPA). Foi fixado um cateter $n$. 24 na veia braquiocefálica e a cadela foi colocada na mesa cirúrgica em decúbito dorsal. $A$ indução anestésica foi realizada com Propofol $(5 \mathrm{mg} / \mathrm{Kg})$, Fentanil $(2 \mathrm{mg} /$ $\mathrm{Kg})$ e Midazolam $(0,15 \mathrm{mg} / \mathrm{Kg})$ por via intravenosa e manutenção com Isoflurano por via inalatória, em fluxo contínuo de oxigênio a $100 \%$ e circuito fechado (Bain). Com objetivo de levar a uma analgesia prolongada no pós-operatório, administrou-se Tramadol $(2,5 \mathrm{mg} / \mathrm{Kg})$ por via subcutânea. Realizou-se tricotomia, antissepsia e foi colocado o pano de campo fenestrado. Após a incisão cutânea e da linha alba, foi realizada a exposição da massa tumoral contida no corpo uterino, em formato esférico (Figura 2). Não havia comprometimento aparente de outros órgãos, apenas uma área de aderência entre a vesícula urinária e o corpo uterino, que foi desfeita. No trans-operatório, devido à descompressão causada pela retirada do útero aumentado, ocorreu uma hipotensão momentânea que foi revertida com a administração de fármacos vasopressores. Em seguida, procedeu-se a ligadura dos pedículos ovarianos e do coto cervical com fio de sutura Vicryl n.2-0 e a remoção de todo o conjunto útero-tubas uterinas-ovários. A sutura da linha alba foi efetuada em cerzidura com nylon n.2-0, seguida da aproximação do subcutâneo em sutura invaginante tipo Cushing e sutura de pele com fio Nylon n.2-0 e pontos tipo Wolf ("U” horizontal) contínuo.
Após a realização do procedimento cirúrgico, o útero com processo tumoral foi pesado $(1,0 \mathrm{~kg}) \mathrm{e}$ encaminhado para histopatologia. A neoformação localizada no corpo do útero $(13,0 \times 10,0 \times 8,0$ $\mathrm{cm})$ tinha aspecto firme, esbranquiçado e sólido. À microscopia com coloração Hematoxilina e Eosina (HE) observou-se: Fragmento de neoformação composta por células fusiformes bem diferenciadas, arranjadas em feixes ondulados com direções aleatórias, exibindo citoplasma eosinofílico, núcleo alongado, central, com cromatina finamente pontilhada. Áreas de necrose coagulativa e vasos sanguíneos foram observados (Figura 3). A coloração Tricrômico de Masson revelou células fusiformes com citoplasma de coloração azulada, indicando origem de tecido conjuntivo fibroso (Figura 4). Diante dos achados microscópicos, concluiu-se o caso como fibroma uterino.

No período pós-operatório, o animal foi tratado com Cefalexina (30mg/kg/VO/BID, por 10 dias), Metronidazol (7,5mg/kg/VO/BID, por 10 dias), Ranitidina (2mg/ $\mathrm{kg} / \mathrm{VO} / \mathrm{BID}$, durante 10 dias) e Tramadol (4mg/kg/VO/BID, por 10 dias). $\mathrm{O}$ animal retornou nos três dias subsequentes à cirurgia para supervisão clínica e administração de medicação endovenosa e retornou após dez dias para retirada dos pontos de pele, verificando-se que apresentava uma excelente recuperação e saúde restabelecida (Figura 5B).

\section{Caso 2}

Foi atendida no ambulatório de pequenos animais do setor de reprodução animal da UNESP-Botucatu, uma cadela, Poodle, de 13 anos de idade, pesando $3,0 \mathrm{~kg}$, nulípara, que apresentava como queixa principal nódulos mamários, com crescimento progressivo, havia aproximadamente dois anos.

Ao exame físico, a cadela apresentava mucosas normocoradas, frequência cardíaca e respiratória dentro dos valores normais de referência, presença de sopro cardíaco e à palpação abdominal, presença de massa consistente, circular, aparentemente em útero. A avaliação das mamas revelou nódulos móveis, não ulcerados, em mama abdominal cranial esquerda $(2 \times 2 \mathrm{~cm}) \mathrm{e}$ torácica esquerda $(2 \times 1 \mathrm{~cm})$. Ao exame ultrassonográfico da cavidade abdominal, visibilizou-se conteúdo uterino e presença de estrutura circular hipoecogênica em útero.

Para realização de hemograma e exames bioquímicos, coletou sangue da jugular, sendo que os achados hematológicos revelaram trombocitose, leucograma inflamatório caracterizado por leucocitose, neutrofilia, eosinofilia e monocitose. Não foram observadas alterações bioquímicas, exceto por um leve aumento da albumina sérica (Tabela 2). 
Tabela 1: Descrição dos parâmetros hematológicos e perfil bioquímico

\begin{tabular}{|c|c|c|}
\hline HEMOGRAMA & VALOR & UNIDADE \\
\hline Hemácias & 2,48 & $10^{6} / \mu \mathrm{L}$ \\
\hline Hemoglobina & 6,10 & $\mathrm{~g} / \mathrm{dL}$ \\
\hline Hematócrito & 20 & $(v g) \%$ \\
\hline${ }^{1} \mathrm{VCM}$ & 80,60 & $f L$ \\
\hline${ }^{2} \mathrm{CHCM}$ & 30,50 & $\%$ \\
\hline${ }^{3} \mathrm{PT}$ & 5,00 & $\mathrm{~g} / \mathrm{dL}$ \\
\hline${ }^{4} \mathrm{RDW}$ & 13,80 & $\%$ \\
\hline Plaquetas & 530.000 & $/ \mu \mathrm{L}$ \\
\hline Fibrinogênio & - & $\mathrm{mg} / \mathrm{dL}$ \\
\hline Metarrubrícitos & 0,00 & $/ 100$ \\
\hline LEUCOGRAMA & VALOR & UNIDADE \\
\hline Leucócitos & 28,8 & $10^{3} / \mu \mathrm{L}$ \\
\hline Mielócitos & 0,0 & $\mu \mathrm{L}$ \\
\hline Metamielócitos & 0,0 & $\mu \mathrm{L}$ \\
\hline Bastonetes & 0,0 & $\mu \mathrm{L}$ \\
\hline Segmentados & 24,5 & $\mu \mathrm{L}$ \\
\hline Linfócitos & 2,6 & $\mu \mathrm{L}$ \\
\hline Eosinófilos & 0,3 & $\mu \mathrm{L}$ \\
\hline Basófilos & 0,0 & $\mu \mathrm{L}$ \\
\hline Monócitos & 1,4 & $\mu \mathrm{L}$ \\
\hline PERFIL BIOQUÍMICO & VALOR & UNIDADE \\
\hline Ureia & 56,0 & $\mathrm{mg} / \mathrm{dL}$ \\
\hline Creatinina & 1,1 & $\mathrm{mg} / \mathrm{dL}$ \\
\hline ALT (TGP) & 36,0 & $\mathrm{UI} / \mathrm{L}$ \\
\hline FA (fosfatase alcalina) & $1.420,0$ & $\mathrm{UI} / \mathrm{L}$ \\
\hline GGT & 36,5 & $\mathrm{UI} / \mathrm{L}$ \\
\hline Proteína total & 3,5 & $\mathrm{~g} / \mathrm{dL}$ \\
\hline Albumina & 1,6 & $\mathrm{~g} / \mathrm{dL}$ \\
\hline Globulina & 1,9 & $\mathrm{~g} / \mathrm{dL}$ \\
\hline
\end{tabular}

${ }^{1} \mathrm{VCM}$ - volume corpuscular médio, ${ }^{2} \mathrm{CHCM}$ - concentração de hemoglobina corpuscular média, ${ }^{3} \mathrm{PT}$ - proteína total (plasma), ${ }^{4} \mathrm{RDW}$ - red cell distribution width.
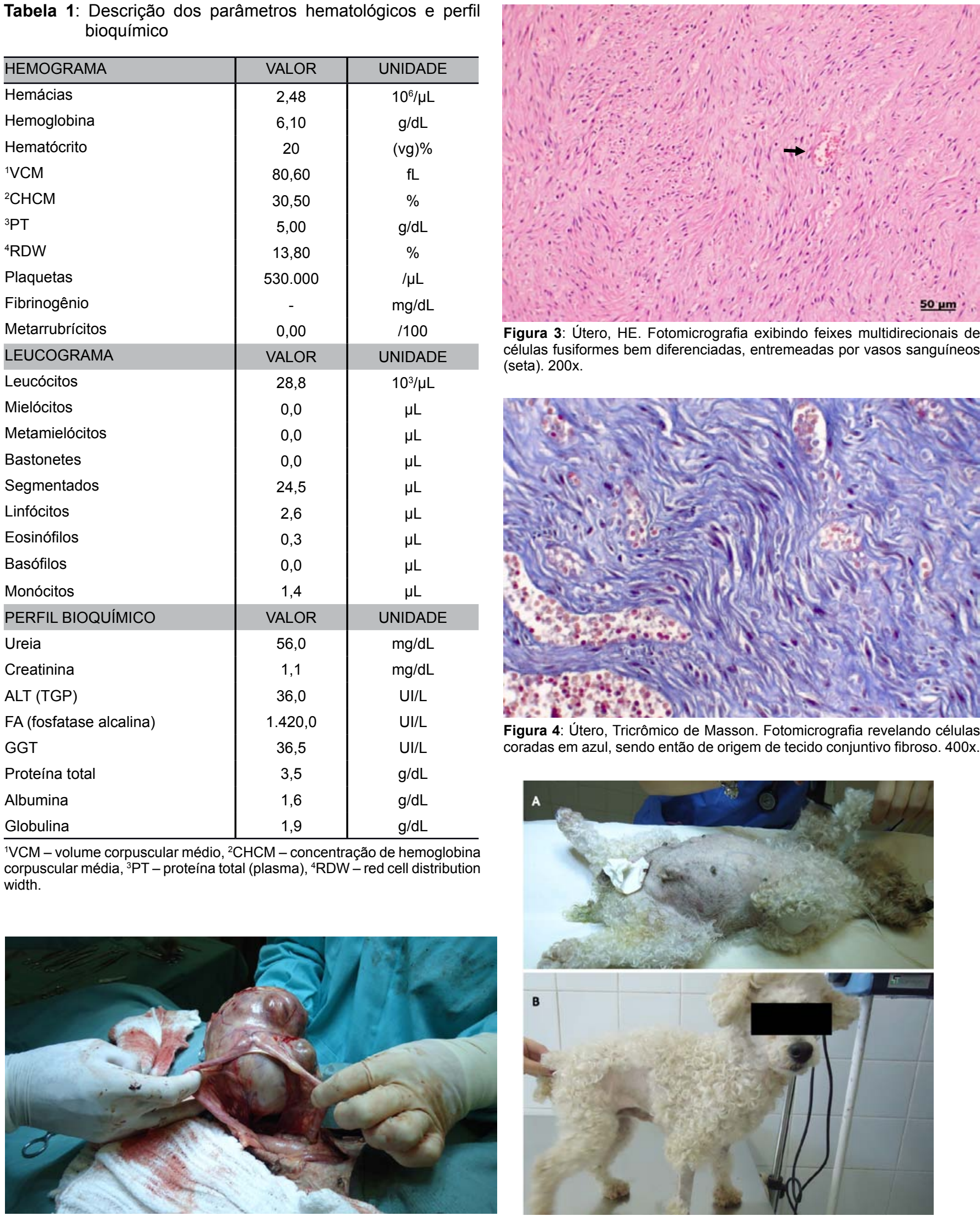

Figura 3: Útero, HE. Fotomicrografia exibindo feixes multidirecionais de células fusiformes bem diferenciadas, entremeadas por vasos sanguíneos (seta). 200x.

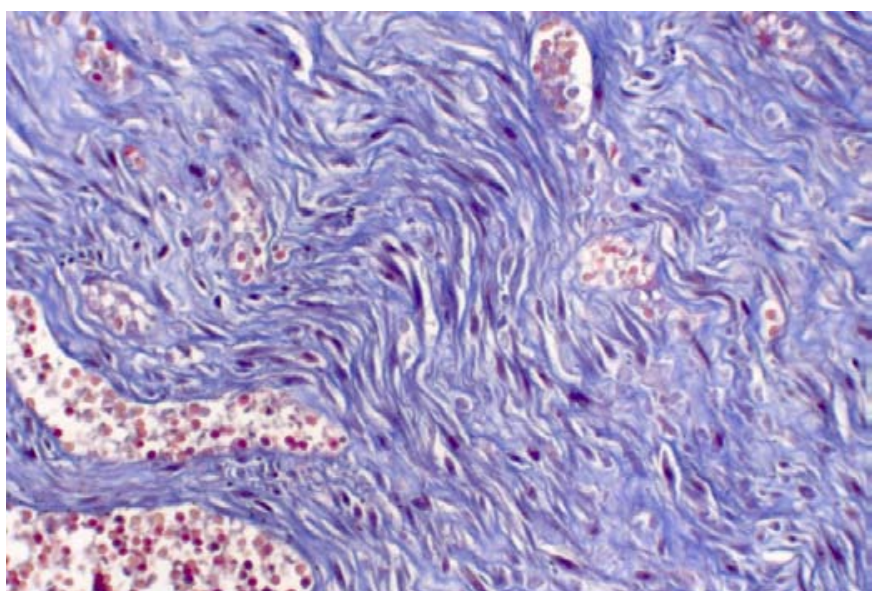

Figura 4: Útero, Tricrômico de Masson. Fotomicrografia revelando células coradas em azul, sendo então de origem de tecido conjuntivo fibroso. 400x.

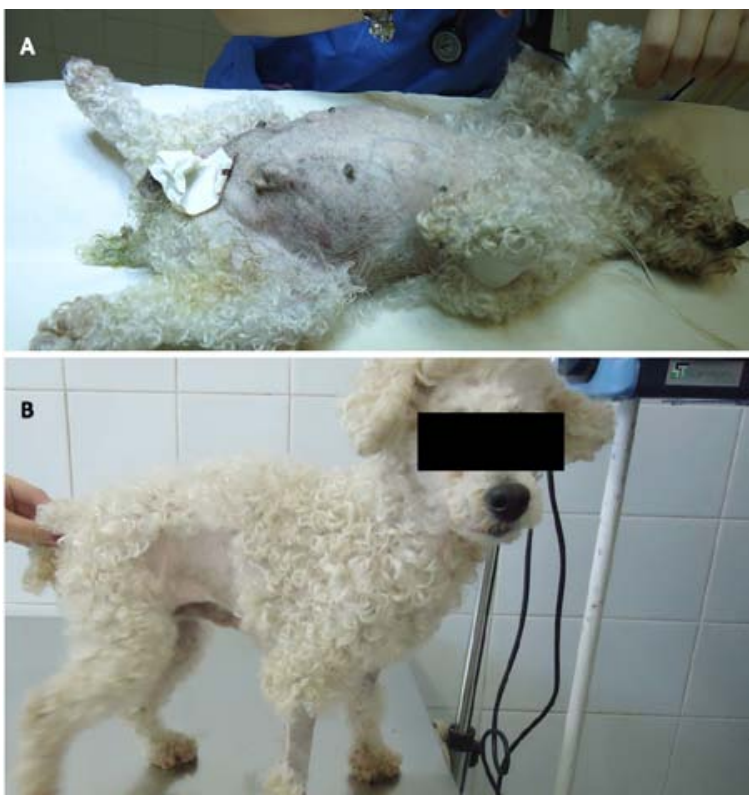

Figura 2: Exposição da massa tumoral contida no corpo uterino, durante o Figura 5A: Primeiro dia de atendimento, animal apresentava abdome procedimento cirúrgico distendido. 5B: Retorno após 10 dias do procedimento cirúrgico. 
Tabela 2: Descrição dos parâmetros hematológicos e perfil bioquímico

\begin{tabular}{|c|c|c|}
\hline HEMOGRAMA & VALOR & UNIDADE \\
\hline Hemácias & 6,31 & $10^{6} / \mu \mathrm{L}$ \\
\hline Hemoglobina & 14,90 & $\mathrm{~g} / \mathrm{dL}$ \\
\hline Hematócrito & 45,00 & $(\mathrm{vg}) \%$ \\
\hline${ }^{1} \mathrm{VCM}$ & 71,30 & $\mathrm{fL}$ \\
\hline${ }^{2} \mathrm{CHCM}$ & 33,10 & $\%$ \\
\hline${ }^{3} \mathrm{PT}$ & 7,00 & $\mathrm{~g} / \mathrm{dL}$ \\
\hline${ }^{4} \mathrm{RDW}$ & 14,50 & $\%$ \\
\hline Plaquetas & 705.000 & $/ \mu \mathrm{L}$ \\
\hline Fibrinogênio & - & $\mathrm{mg} / \mathrm{dL}$ \\
\hline Metarrubrícitos & 0,00 & $/ 100$ \\
\hline LEUCOGRAMA & VALOR & UNIDADE \\
\hline Leucócitos & 19,20 & $10^{3} / \mu \mathrm{L}$ \\
\hline Mielócitos & 0,00 & $\mu \mathrm{L}$ \\
\hline Metamielócitos & 0,00 & $\mu \mathrm{L}$ \\
\hline Bastonetes & 0,00 & $\mu \mathrm{L}$ \\
\hline Segmentados & 14,60 & $\mu \mathrm{L}$ \\
\hline Linfócitos & 1,30 & $\mu \mathrm{L}$ \\
\hline Eosinófilos & 1,50 & $\mu \mathrm{L}$ \\
\hline Basófilos & 0,00 & $\mu \mathrm{L}$ \\
\hline Monócitos & 1,70 & $\mu \mathrm{L}$ \\
\hline PERFIL BIOQUÍMICO & VALOR & UNIDADE \\
\hline Ureia & 30,00 & $\mathrm{mg} / \mathrm{dL}$ \\
\hline Creatinina & 0,50 & $\mathrm{mg} / \mathrm{dL}$ \\
\hline ALT (TGP) & 72,00 & $\mathrm{UI} / \mathrm{L}$ \\
\hline FA (fosfatase alcalina) & 35,00 & $\mathrm{UI} / \mathrm{L}$ \\
\hline GGT & 2,20 & UI/L \\
\hline Proteína total & 6,90 & $\mathrm{~g} / \mathrm{dL}$ \\
\hline Albumina & 3,60 & $\mathrm{~g} / \mathrm{dL}$ \\
\hline Globulina & 3,30 & $\mathrm{~g} / \mathrm{dL}$ \\
\hline
\end{tabular}

${ }^{1}$ VCM - volume corpuscular médio, ${ }^{2} \mathrm{CHCM}$ - concentração de hemoglobina corpuscular média, ${ }^{3} \mathrm{PT}$ - proteína total (plasma), ${ }^{4} \mathrm{RDW}$ - red cell distribution width.

Diante do quadro clínico, foi realizada OSH de caráter emergencial. O animal foi sedado com Morfina $(0,8 \mathrm{mg} / \mathrm{kg})$ por via intramuscular, após 10 minutos foi fixado um cateter $n$. 24 na veia braquiocefálica e a cadela foi colocada na mesa cirúrgica em decúbito dorsal. A indução anestésica foi realizada com Propofol $(5 \mathrm{mg} / \mathrm{Kg})$, Fentanil $(2 \mathrm{mg} / \mathrm{Kg})$ e Midazolam $(0,15 \mathrm{mg} / \mathrm{Kg})$ por via intravenosa e manutenção com Isoflurano por via inalatória, em fluxo continuo de oxigênio a $100 \%$ e circuito fechado (Bain).

Para efetuar o procedimento cirúrgico, realizou-se tricotomia, antissepsia e foi colocado o pano de campo fenestrado. Após a incisão cutânea e da linha alba, foi realizada a exposição do útero contendo uma massa firme em região de corpo (Figura 6). Em seguida, procedeu-se à ligadura dos pedículos ovarianos e do coto cervical com fio de sutura Vicryl n. 2-0 e a remoção do útero, tubas uterinas e ovários. A sutura da linha alba foi efetuada em cerzidura com náilon n. 2-0. Neste mesmo procedimento efetuou-se nodulectomia nas duas mamas com neoformação. Para aproximação do subcutâneo, utilizou-se sutura invaginante tipo Cushing e sutura de pele com fio náilon n. 2-0 e pontos tipo Wolf ("U" horizontal) contínuo.

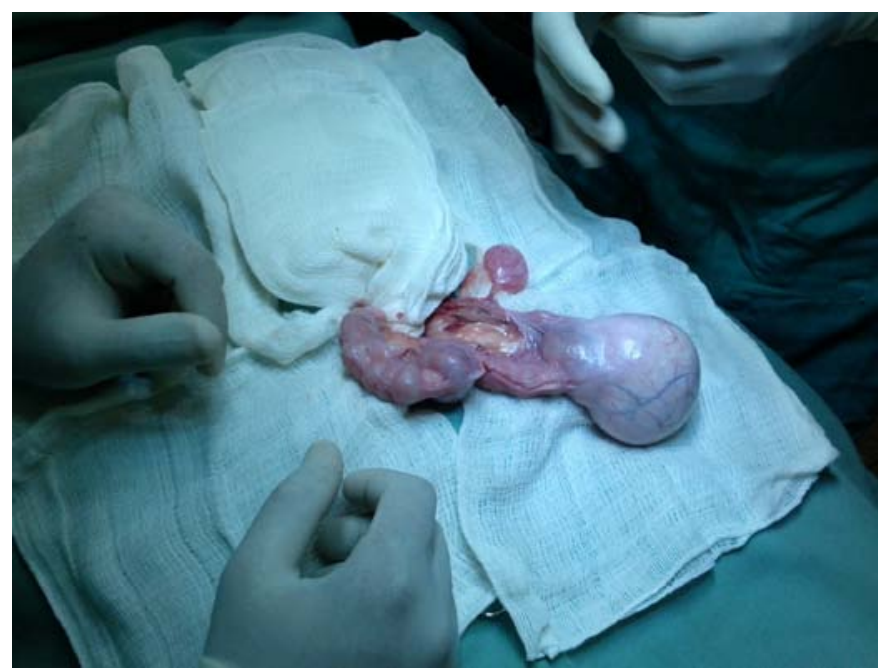

Figura 6. Útero contendo fibroma em região de corpo.

O útero com processo tumoral arredondado, firme, medindo $7,0 \times 7,0 \times 6,5 \mathrm{~cm}$ foi encaminhado para histopatologia. $\grave{A}$ microscopia com coloração Hematoxilina e Eosina (HE) observou-se: proliferação de células fusiformes, arranjadas em cordões e feixes, sustentadas por estroma denso colagenoso, com focos de neovascularização. As células fusiformes apresentavam citoplasma fibrilar de aspecto ondulado eosinofílico de limites indistintos. O núcleo era alongado e por vezes arredondado, cromatina finamente agregada, nucléolo único e por vezes proeminente, além de anisocariose discreta. Concluiu-se o caso como fibroma.

Para o tratamento pós-operatório foram receitados Cefalexina (30 mg/Kg/BID/VO), Meloxicam (0,1 mg/Kg/SID/VO), Tramadol (4mg/Kg/BID/VO), Dipirona (1 gota/kg/TID/VO), Omeprazol $(1,0 \mathrm{mg} / \mathrm{kg} / \mathrm{SID} / \mathrm{VO})$, além de Rifamicina para administração tópica. O animal retornou após 10 dias para reavaliação e retirada dos pontos de pele, encontrando-se com a saúde restabelecida.

\section{Discussão e conclusão}

As neoplasias uterinas são infrequentes em cadelas; além disso, por tratar-se de uma patologia hormônio-dependente, fêmeas idosas não castradas são mais propensas a serem acometidas (Souza et al., 2012), o que se confirmou nestes relatos.

Em um estudo retrospectivo de 43 casos de neoplasias mesenquimais em genitálias de cadelas (útero, cérvix, vagina e vulva), observou-se $44,2 \%$ de tumores vaginais, $27,9 \%$ uterinos, $23,3 \%$ vulvares e $4,6 \%$ de tumores em cérvix sendo que, destes, $46,5 \%$ (20/43) eram leiomiomas, $41,9 \%$ (18/43) fibroleiomiomas, $7,0 \%(3 / 43)$ fibromas e $4,6 \%(2 / 43)$ leiomiossarcomas. Entretanto, nesta retrospectiva, não foi relatado nenhum fibroma uterino, demonstrando a baixa incidência deste tipo de tumor 
em útero (Souza et al., 2012). Porém, frente ao presente relato de dois casos de fibroma uterino em cadelas, diagnosticados num período em que nenhum animal atendido foi diagnosticado com leiomioma uterino, é importante que a elaboração de um novo levantamento seja considerada.

Dentre os sinais clínicos presentes no caso 1 , a distensão abdominal, a anorexia e o tenesmo justificam-se pela compressão causada pela massa tumoral nos órgãos adjacentes, visibilizada nos exames radiográfico e ultrassonográfico, semelhante às observações de Sontas et al. (2010).

O diagnóstico foi realizado por meio dos exames físicos, auxiliados pela radiografia e ultrassonografia e o diagnóstico definitivo do tipo celular envolvido adveio do exame histopatológico da peça cirúrgica, extremamente importante para a conduta pósoperatória.

Verificou-se que a coloração de Tricrômico de Masson é muito importante na diferenciação entre tecido muscular e colagenoso, além de apresentar baixo custo, o que facilita seu emprego na rotina de diagnóstico de patologia veterinária para diferenciar as classificações tumorais, uma vez que pode ser difícil caracterizá-

\section{Referências}

BABA, A.I.; CÂTOI, C. Comparative Oncology. Bucharest: The Publishing House of the Romanian Academy, 2007.

KLEIN, M.K. Tumors of the female reproductive system. In: WITHROW, S.J.; MacEWEN E.G. (Eds.). Small Animal Clinical Oncology. Philadelphia: W.B. Saunders, p. 445-454, 2001.

MacLACHLAN, N.J.; KENNEDY, P.C.Tumors of the genital system. In: D.J. Meuten, (Ed.). Tumors in Domestic Animals, lowa: lowa State Press, 2002.

MANAROLLA, G.; CASEIRO, S.; SIRONI, G.; RAMPIN, T. Morphological and immunohistochemical observations on leiomyoma of the ventral ligament of oviduct of the hen. J. Comp. Pathol, v.144, p.180-186, 2011.

McENTEE, M.C. Reproductive Oncology. Clinical Techniques in Small Animal Practice, v. 17, n. 3, p. 133-149, 2002.
Ios apenas pela Hematoxilina \& Eosina (HE) conforme também constatado por Munday e Stedman (2002) e Manarolla et al. (2011).

Os achados de hematologia e bioquímica sérica foram condizentes com o quadro clínico do animal e semelhantes aos relatados por Sontas et al. (2010) em leiomioma uterino numa cadela. Podemos escalonar os casos em relação à gravidade, por isso os achados hematológicos e bioquímicos dos dois casos foram diferentes. Observamos que o caso 2 foi um achado ocasional, diagnosticado através da realização de um bom exame clínico e o caso 1 a queixa principal estava relacionada diretamente ao caso.

Diante do quadro clínico, nos dois casos não houve outra opção a não ser o tratamento cirúrgico, recomendado pela literatura consultada (Sontas et al., 2010), sendo este executado em caráter emergencial, devido ao estado geral dos pacientes e risco de agravamento, podendo progredir a óbito.

Pela pouca frequência do caso clínico, esse relato torna-se relevante para embasar os atendimentos ambulatoriais em reprodução de pequenos animais, visto que o tratamento executado resultou em sucesso em ambos os casos.

MUNDAY, J.S.; STEDMAN, N.L. Uterine leiomyomas in two Vietnamese Pot-bellied pigs (Sus scrofa). Vet. Pathol, v. 39, p. 580-583, 2002.

SCHLAFER, D.H.; MILLER, R.B. Female genital system. In: Maxie M.G. (Ed.), Jubb, Kennedy and Palmer's Pathology of Domestic Animals. 5th ed. Philadelphia: Elsevier, 2007, p. 429564.

SONTAS, B.H., OZYOGURTCU, H.; TURNA, O.; ARUN, S.; $\mathrm{EKICl}, \mathrm{H}$. Uterine Leiomyoma in a Spayed Poodle Bitch: A Case Report. Reprod Dom Anim v. 45, p. 550-554, 2010.

SOUZA, S.O.; WATANABE, T.T.N.; CASAGRANDE, R.A.; WOUTERS, A.T.B.; WOUTERS, F.; DRIEMEIER, D.

Histopathological and immunohistochemical characterization of mesenchymal neoplasms of the genitalia in 43 bitches. Pesquisa Veterinária Brasileira v. 32, n.12), p. 1313-1318, 2012. 